\title{
Características de los sujetos-objetos en la producción científica de química avanzada: el caso del CIMAV, México
}

Characteristics of subjects-objects in the scientific production of advanced chemistry: the case study of the CIMAV (Mexico)

\author{
Rocío ANCHONDO-Granados, Javier TARANGo, Gerardo Ascencio-BACA \\ Universidad Autónoma de Chihuahua, Facultad de Filosofía y Letras, Campus Universitario \#1 Chihuahua, Chih., México 31130, \\ Apartado Postal 744, roxioag@gmail.com, tj.88888@hotmail.com, gbaca@uach.mx
}

\begin{abstract}
Resumen
Se aplica un conjunto de indicadores bibliométricos para la evaluación de un centro de investigación público en México, el Centro de Investigación en Materiales Avanzados (CIMAV), especializado en química avanzada; y posteriormente, se desarrolla una comparación de resultados con los lineamientos que definen las características y condiciones en que debiera suceder la comunicación científica.
\end{abstract}

Palabras clave: Producción científica. Evaluación. Química. Méjico. Centro de Investigación en Materiales Avanzados (CIMAV).

\section{Introducción}

La constante en el desarrollo de procesos de comunicación científica es el reflejo del crecimiento de las instituciones a través de sus investigadores (sujetos) y sus publicaciones (objetos). Todo ello, tomando como referencia que tales comportamientos estén alineados a un conjunto de normas y reglamentaciones, de tal manera que alcancen la suficiencia en calidad y cantidad, y que, además, sea posible la toma de decisiones a través de su evaluación.

En el caso de México, debe considerarse que no existen normas específicas que identifiquen y diferencien niveles de competitividad individual y colectiva, observándose un matiz amplio de opciones que van desde visiones institucionales internas hasta propuestas nacionales.

La normatividad nacional más aceptada para evaluar la comunicación científica es la propuesta por el Sistema Nacional de Investigadores (SNI), dependiente del Consejo Nacional de Ciencia y Tecnología (CONACYT), donde se especifica lo siguiente (CONACYT, 2014; UNAM, 2013). De forma general, valora artículos indizados (Nivel A incluidos en ISI, en el catálogo de revistas de CONACYT y recientemente en Scopus); artículos arbitrados publicados en revistas de Nivel B (incluidos en otros

\begin{abstract}
A set of bibliiometric indicators is applied for the evaluation of a public research center in Mexico, the Research Center in Advanced Materials (CIMAV), specialized in advanced Chemistry; and then the results are compared with the guidelines that define the characteristics and conditions in which scientific communication should develop.
\end{abstract}

Keywords: Scientific production. Evaluation. Chemistry. Mexico. Research Center in Advanced Materials (CIMAV).

medios); autoría de primeros autores, por institución, colaboraciones nacionales e internacionales y citas; grados académicos (doctorado); y niveles de acreditación de los programas académicos en los que imparte docencia. De forma específica para la disciplina química se consideran, además de lo anterior, la publicación de productos en una línea temática específica; artículos incluidos en Citation Index; niveles de impacto a través de citaciones; desarrollos tecnológicos, proyectos de investigación, tesis dirigidas (preferentemente de posgrado); y desarrollo de patentes.

\section{Delimitación del estudio}

En México existen 27 centros públicos de investigación (dependientes de CONACYT), los cuales, para su evaluación, son los que más se aproximan a los requerimientos normativos antes mencionados. Tales instituciones están distribuidas geográficamente en el país según necesidades específicas que determinan sus líneas de investigación (Rodríguez-Rodríguez, 2013). Sus objetivos principales son: divulgar en la sociedad, la ciencia y la tecnología; innovar en la generación, desarrollo, asimilación y aplicación del conocimiento en ciencia y tecnología; entre otros (SEP, 2013). 
Este estudio toma como referencia un caso: el Centro de Investigación en Materiales Avanzados (CIMAV), ubicado en Chihuahua, México, considerando para su elección que es uno de los 27 centros públicos de investigación CONACYT en México, el primero en su clase en el estudio de la química avanzada (materiales) y con altos índices de competitividad científicoeducativa en producción, calidad e innovación (Rodríguez-Rodríguez, 2013).

\section{Objetivos}

Se plantean los siguientes objetivos:

- Recurriendo a los estudios métricos tradicionales, identificar características de los sujetos y objetos relacionados con la comunicación científica de una disciplina particular.

- De acuerdo con los resultados, comparar tales características con distintos indicadores, procurando identificar niveles de competitividad.

- Derivar recomendaciones generales que propicien elementos de mejora institucional en la manera como suceden los procesos de comunicación científica.

\section{Metodología}

Esta investigación tiene las siguientes características:

- Unidad de análisis y observación: Total de artículos publicados durante el período 20062011 por 40 investigadores del CIMAV.

- Fuentes utilizadas: bases de datos de ISI, Scopus, Science Direct y RedALyC.

- Variables e indicadores: Compuesto por seis grupos, mismos que al recurrir a modelos métricos tradicionales, se justifican en su aproximación a los lineamientos de evaluación en México: 1) características de la publicación; 2) modelo de Price (vida media, tasa de envejecimiento y referencias operativas y de archivo); 3) modelo de Bradford (concentración-dispersión de la literatura científica); 4) modelo de Zipf sobre frecuencias de palabras clave utilizadas; 5) modelo de Lotka (identificación de las élites intelectuales e indicadores de colaboración, en particular la proporción de documentos coautorados, índice de coautoría y grado de colaboración, distribución geográfica e institucional); y 6) indicadores de impacto, citas recibidas, factor de impacto $(\mathrm{FI})$ de las revistas, $\mathrm{FI}$ de 5 años, índice de inmediatez del ISI
Web of Knowledge y Source-Normalized Impact per Paper (SNIP).

\section{Análisis de resultados}

Los datos recolectados corresponden a 667 artículos científicos, los cuales representan el universo de la producción del período de evaluación (2006-2011), mismos que observan un crecimiento longitudinal, correspondiendo 79 al primer año de evaluación y 135 en el último, lo cual implica un crecimiento porcentual del $58.51 \%$ en cinco años. Según los resultados del último año evaluado, el promedio de artículos publicados por investigador es de 2.25 sin especificar orden de autorías.

El tipo de revistas en donde se publicaron tales artículos, fueron evaluadas bajo cuatro criterios:

- Arbitraje: El 98.5\% se incluyeron en revistas con arbitraje y sólo $1.49 \%$ en revistas sin arbitraje.

- Indización en bases de datos: 545 artículos se publicaron en revistas indizadas que aparecen tanto en ISI como en Scopus; 37 artículos en revistas indizadas solamente en ISI; 32 artículos sólo en Scopus; y 46 artículos aparecieron en revistas no indizadas en las principales bases de datos.

- Circulación: 650 artículos fueron publicados en revistas de circulación internacional (98\%) y sólo 10 artículos en revistas de circulación nacional $(2 \%)$.

- Especialización: 634 artículos se publicaron en revistas especializadas de las ciencias químicas (96\%) y 26 artículos en revistas no especializadas o de otra disciplina (4\% restante).

Para medir el crecimiento y envejecimiento de la literatura científica publicada, se utilizó el modelo de Price y se recurrió a las referencias de los 667 artículos identificados. En relación a la vida media el resultado global fue de 8.08. Como consecuencia de lo anterior, los artículos evaluados observaron una tasa de envejecimiento promedio de 91.77 , significando esto que, ante el crecimiento en la producción científica del área de la química avanzada, los contenidos publicados dejaron de ser útiles en muy corto tiempo, evitándose en consecuencia mayor número de citaciones.

La clasificación de las referencias (operativas y de archivo) indicaron que sólo el $28.8 \%$ promedio fueron operativas (con menos de cinco años de antigüedad) y las mayores a cinco años, conocidas como referencias de archivo, corres- 
pondieron al $71.2 \%$, representando un alto nivel de ruido cronológico.

Respecto al indicador de concentración-dispersión, obtenido a través del modelo de Bradford, resultó que el total de los artículos aparecieron publicados en 266 revistas científicas, identificándose posteriormente cuántas revistas tenían $n$ artículos. Además de las frecuencias de artículos, a través de este modelo, se permitió dividir los títulos de las revistas en tres zonas: mayor concentración (199 artículos en 15 títulos), concentración media (201 artículos en 48 títulos) y baja concentración o dispersión (260 artículos en 203 títulos de revistas).

Según los resultados anteriores, la totalidad de revistas científicas en las que se publicaron los artículos evaluados fue de 323, siendo el $62.84 \%$ de la zona de baja concentración, $14.86 \%$ en la de mediana concentración y $4.64 \%$ en la de mayor concentración.

De las 15 revistas que componen la zona de mayor concentración, tres no identifican datos de FI; y 13 sí lo hacen (rango menor de 0.300 y mayor de 3.903). En relación con el idioma, 13 publican en inglés y dos en español. Respecto a la distribución geográfica, dos son nacionales y el resto internacionales.

Para comprobar las agrupaciones temáticas, se utilizó el modelo de Zipf a través del conteo de las palabras clave incluidas en los artículos examinados. Se tomaron en cuenta hasta 10 palabras clave, observándose como resultado 5,399 palabras solas o compuestas, de las cuales sólo 1,527 eran diferentes. La palabra clave que mayor frecuencia observó fue "Propierties" con 64 apariciones y "Materials" con 52 en la tercera posición —término que la institución considera prioritario-. 804 términos se registraron sólo una vez.

Respecto a la productividad de los autores científicos y la identificar élites intelectuales, se utilizó el modelo de Lotka, con el fin de identificar rasgos de comportamiento en la productividad de artículos, tanto de forma individual como colectiva. Los resultados recolectados corresponden solamente a los autores institucionales (no comprende estudiantes, tesistas, técnicos, investigadores externos, etc.). Los resultados obtenidos a través de la aplicación de este modelo fueron:

- Identificación de la élite intelectual: De 98 autores identificados, solamente 9.8 (redondeado a 10) de 40 pertenecen a la élite intelectual, que aportó 418 contribuciones, representando el $36.89 \%$ del total de las contribuciones.
- Indicadores de colaboración: El índice de coautoría arrojó 5.24 autores promedio por artículo; y el grado de colaboración (expresado entre 0 y 1) marcó 0.98 .

- Distribución geográfica de la colaboración: En el ámbito internacional los países con los que más se ha colaborado son Estados Unidos (100 contribuciones), España (97 contribuciones) y Francia (con 54 colaboraciones).

- Distribución institucional de la colaboración: Este indicador identifica la existencia de colaboración institucional con 33 instituciones, de las cuales 26 son nacionales $(78.78 \%)$ y siete internacionales $(21.21 \%)$. Las tres principales instituciones de colaboración son el Centro de Investigación y Estudios Avanzados del Politécnico Nacional, UNAM y Universidad Autónoma de Chihuahua (UACH), representando el $24.1 \%$ del total en las tres instituciones nacionales.

En lo que se refiere a los indicadores de impacto, los datos se basaron en los conteos de citas y la identificación de cálculos que realizan las principales bases de datos (Web of Science WOS- de ISI y Scopus) cuyos resultados fueron:

- Citas en WOS: El 30.6\% de los artículos publicados no recibieron citas, mientras que el $48.8 \%$ recibieron entre una y cinco citas. En el extremo se muestra que siete artículos han recibido 26 ó más citas, representando un $1.2 \%$ de la producción total. Los artículos publicados obtuvieron 2,060 citas, que dividido entre el total de artículos promedió 3.12 citas en WOS por articulo.

- Citas en Scopus: En esta base de datos se identificaron 575 artículos, en donde el $29.6 \%$ de los artículos publicados no recibieron citas, mientras que el $49.2 \%$ recibieron entre 1 y 5 citas. En el extremo se identificó que 12 artículos han recibido 26 ó más citas (2.1\% de la producción total). Los artículos científicos publicados durante el periodo de cinco años obtuvieron 2,235 citas, que dividido entre el total de artículos contemplados para este estudio, nos da un promedio de 3.39 citas en Scopus por artículo.

La evaluación del FI fue obtenido a partir de la consulta del Journal Citation Report (JCR), por lo que sólo se consideraron las revistas indizadas en ISI. El promedio general fue de 2.087 con una desviación estándar 1.485. La mayoría de los investigadores publican artículos en revistas que tienen un FI de hasta de 2.000 con 159 artículos en 69 títulos registrados $(32.1 \%$ del total de títulos). 
Además, se elaboró un cálculo de $\mathrm{FI}$ de cinco años que aparecen en publicaciones indizadas en ISI. El resultado promedio fue de 2.176 con una desviación estándar de 1.505. La mayoría de los artículos se publicaron en revistas con FI de 5 años de hasta 3.000 , con 162 artículos publicados $(29.8 \%$ del total).

Otro indicador evaluado es el Índice de Inmediatez a partir del JCR, por lo que sólo aparecen revistas indizadas en ISI. Se calculó el promedio del Índice de Inmediatez y se obtuvo un resultado de 0.384 , con una desviación estándar de 0.324 . Los resultados obtenidos observan que la mayoría de los artículos publicados aparecen en 200 títulos de revistas que tienen un Índice de Inmediatez promedio de 2.000 (94.8\% del total) y 508 artículos (96.6\%).

En el caso del SNIP, este fue calculado de acuerdo a la información recolectada en Scopus, por lo que únicamente comprendió revistas indizadas en dicha base de datos. Se calculó el promedio del SNIP y se obtuvo un resultado de
1.161 con una desviación estándar de 0.844 . Los resultados de la producción científica en este indicador se caracteriza al oscilar entre 0.000 y 0.999 , con 123 títulos (53.5\% del total) y 297 artículos (50.3\%).

\section{Características e indicadores: análisis comparativo}

A manera de interpretación de los resultados antes presentados, enseguida se muestra una comparación entre los indicadores evaluados y los aspectos normativos y lineamientos que rigen en México la evaluación de la producción científica, con lo cual pudiera hacerse más visible las condiciones que suceden en la entidad y disciplina evaluadas. La totalidad de los indicadores fueron agrupados en tres conglomerados:

\subsection{Características de la publicaciones}

Comprende ocho indicadores relacionados con aspectos que distinguen la forma como suceden las publicaciones (Tabla I).

\begin{tabular}{|c|c|c|}
\hline Indicador & Análisis comparativo & Consideraciones de valor \\
\hline $\begin{array}{l}\text { Número } \\
\text { de artículos } \\
\text { promedio por año } \\
\text { por investigador }\end{array}$ & $\begin{array}{l}\text { SNI indica que para la disciplina de las ciencias } \\
\text { químicas, cada investigador, aunque } \\
\text { dependiendo del nivel de evaluación (Nivel I, III y } \\
\text { III), se espera como mínimo un artículo como } \\
\text { primer autor por año en promedio. }\end{array}$ & $\begin{array}{l}\text { El resultado observado es un promedio de } 2.25 \text { artículos } \\
\text { por autor por año, sin especificar si aparecen como } \\
\text { primeros autores. }\end{array}$ \\
\hline Arbitraje & $\begin{array}{l}\text { Para la disciplina de ciencias químicas en } \\
\text { general, el SNI no valora las publicaciones sin } \\
\text { arbitraje estricto. }\end{array}$ & $\begin{array}{l}\text { El } 98.5 \% \text { de los artículos fueron publicados en revistas } \\
\text { con arbitraje estricto. Se propone alcanzar el } 100 \% \text {. }\end{array}$ \\
\hline Indización & $\begin{array}{l}\text { El lineamiento es claro al especificar que en el } \\
\text { área química son considerados de alto valor sólo } \\
\text { artículos indizados en ISI, Catalogo de Revistas } \\
\text { de CONACYT y Scopus. }\end{array}$ & $\begin{array}{l}\text { El } 88.58 \% \text { de los resultados corresponden a artículos } \\
\text { indizados, siendo la recomendación alcanzar el } 100 \% \text {. }\end{array}$ \\
\hline Circulación & $\begin{array}{l}\text { De forma tácita, en México se valora mejor a las } \\
\text { publicaciones en el extranjero, especialmente en } \\
\text { idioma inglés. }\end{array}$ & $\begin{array}{l}\text { El } 98 \% \text { de las publicaciones fueron hechas en el } \\
\text { extranjero. Este indicador se deberá justificar siempre y } \\
\text { cuando las publicaciones sucedan en revistas indizadas } \\
\text { sin importar el país. }\end{array}$ \\
\hline $\begin{array}{l}\text { Especialización } \\
\text { y líneas temáticas }\end{array}$ & $\begin{array}{l}\text { Los centros públicos de investigación en México } \\
\text { fueron creados ex profeso para cubrir } \\
\text { necesidades específicas, tanto geográficas } \\
\text { como disciplinares, se tiene la expectativa de } \\
\text { que la totalidad de los artículos publicados } \\
\text { correspondan a la disciplina general que } \\
\text { identifica al centro de investigación. }\end{array}$ & $\begin{array}{l}\text { 96\% de las publicaciones evaluadas correspondieron a } \\
\text { química avanzada. En relación con las palabras clave, } \\
\text { se observa alta concentración en aquellas de interés } \\
\text { institucional con alta dispersión en porcentajes elevados } \\
\text { que no lo son. Se recomienda lograr la especialización } \\
\text { demandada al } 100 \% \text { y buscar medios de concentración } \\
\text { de temáticas específicas. }\end{array}$ \\
\hline $\begin{array}{l}\text { Distribución } \\
\text { geográfica } \\
\text { de la colaboración }\end{array}$ & $\begin{array}{l}\text { El deseo implícito de los procesos de evaluación } \\
\text { en México, propone el acercamiento a países } \\
\text { cuyos niveles de desarrollo sean superiores al } \\
\text { propio. }\end{array}$ & $\begin{array}{l}\text { Los resultados muestran mayores índices de } \\
\text { acercamiento con Estados Unidos, España y Francia, } \\
\text { todos líderes en su propio idioma. Este indicador no } \\
\text { puede ser valorado de forma precisa. }\end{array}$ \\
\hline $\begin{array}{l}\text { Distribución } \\
\text { institucional } \\
\text { de la colaboración }\end{array}$ & $\begin{array}{l}\text { El deseo implícito de los procesos de evaluación } \\
\text { en México, propone el acercamiento a } \\
\text { instituciones internacionales de alto } \\
\text { reconocimiento. }\end{array}$ & $\begin{array}{l}\text { Los resultados muestran mayor colaboración con } \\
\text { instituciones nacionales }(78.78 \%) \text {. Sin embargo este } \\
\text { indicador no puede ser valorado de forma precisa. }\end{array}$ \\
\hline $\begin{array}{l}\text { Indicadores } \\
\text { de colaboración } \\
\text { de autores }\end{array}$ & $\begin{array}{l}\text { Aunque los parámetros del SNI en cuestiones de } \\
\text { colaboración son poco precisos, indican la } \\
\text { necesidad de publicar, cuando menos por año, } \\
\text { un artículo de autoría individual o como primer } \\
\text { autor, así como un máximo recomendado de tres } \\
\text { autorías por artículo. }\end{array}$ & $\begin{array}{l}\text { Se recomienda reorientar el número de autorías por } \\
\text { artículo, ya que excede en mucho lo recomendado con } \\
\text { un promedio de } 5.24 \text { y un número ínfimo de artículos con } \\
\text { de autoría única. }\end{array}$ \\
\hline
\end{tabular}

Tabla I. Características de las publicaciones 


\subsection{Indicadores bibliométricos básicos}

Comprende cuatro indicadores enfocados principalmente a la consistencia interna de los documentos publicados (Tabla II).

\begin{tabular}{|c|c|c|}
\hline Indicador & Análisis comparativo & Consideraciones de valor \\
\hline $\begin{array}{l}\text { Vida media y tasa } \\
\text { de envejecimiento }\end{array}$ & $\begin{array}{l}\text { De estos dos indicadores no se encontró un punto de } \\
\text { referencia concreta. En el caso de la química, física y } \\
\text { matemáticas, en México de manera informal, se toleran } \\
\text { cinco años de vida media y } 80 \text { puntos de tasa de } \\
\text { envejecimiento. }\end{array}$ & $\begin{array}{l}\text { Los resultados observados fueron: vida media } \\
\text { ( } 8.08 \text { años) y tasa de envejecimiento de } 91.77 \text {. } \\
\text { Ambos resultados demuestran baja permanencia } \\
\text { de los contenidos provocados por el uso de } \\
\text { referencias obsoletas. }\end{array}$ \\
\hline $\begin{array}{l}\text { Referencias } \\
\text { operativas } \\
\text { y de archivo }\end{array}$ & $\begin{array}{l}\text { Aunque no existe una normativa legitimada en este } \\
\text { indicador, se sabe de manera informal, que en las } \\
\text { ciencias químicas la tolerancia es de hasta } 20 \% \text { de } \\
\text { referencias de archivo y } 80 \% \text { de referencias operativas. } \\
\text { Se ha manifestado incluso una tolerancia promedio } \\
\text { general de hasta el } 50 \% \text { y } 50 \% \text {. }\end{array}$ & $\begin{array}{l}\text { Los resultados generales del } 28.8 \% \text { de referencias } \\
\text { operativas y } 71.2 \% \text { de referencias de archivo } \\
\text { ofrece resultados no favorables que demandan } \\
\text { atención. La recomendación se relaciona con el } \\
\text { uso de fuentes de información con menor } \\
\text { obsolescencia. }\end{array}$ \\
\hline $\begin{array}{l}\text { Concentración- } \\
\text { Dispersión }\end{array}$ & $\begin{array}{l}\text { De este indicador no se identificó un parámetro } \\
\text { concreto de comparación. De forma precisa se } \\
\text { esperaría que la concentración correspondiera a las } \\
\text { revistas de mayor prestigio en la disciplina estudiada. }\end{array}$ & $\begin{array}{l}\text { Se recomienda que el } 100 \% \text { de los núcleos de } \\
\text { concentración incluyan publicaciones con } \mathrm{FI}, \\
\text { buscando mejorar sus niveles promedio, ya que } \\
\text { no sobrepasa el } 3.000 \text { y un par de publicaciones } \\
\text { no incluyen este indicador métrico. }\end{array}$ \\
\hline Élite intelectual & $\begin{array}{l}\text { No se identificó parámetro que determine la } \\
\text { composición ideal de las élites intelectuales. }\end{array}$ & $\begin{array}{l}\text { Se infiere la necesidad de que se promueva la no } \\
\text { diferenciación sustancial entre investigadores en } \\
\text { el número de artículos publicados. La cantidad de } \\
\text { artículos publicados depende de múltiples } \\
\text { factores. }\end{array}$ \\
\hline
\end{tabular}

Tabla II. Indicadores bibliométricos básicos

\subsection{Indicadores de impacto}

Se compone de tres indicadores que se relacionan con la calidad del documento y su influencia en la generación de nuevo conocimiento (Tabla III).

\begin{tabular}{|c|c|c|}
\hline Indicador & Análisis comparativo & Consideraciones de valor \\
\hline Citación & $\begin{array}{l}\text { Existe imprecisión en este indicador, ya que los } \\
\text { lineamientos de SNI consideran, al menos, } 350 \\
\text { citas relacionadas a lo largo de la vida del } \\
\text { investigador. }\end{array}$ & $\begin{array}{l}\text { Los resultados indican que el WOS y en Scopus el } 30.6 \% \text { y } \\
29.6 \% \text { respectivamente no recibieron ninguna cita. Si el } \\
\text { promedio de citas fue de } 3.12 \text { en WOS y de } 2.235 \text { en Scopus, } \\
\text { para alcanzar el parámetro de SNI se requieren al menos } 12 \\
\text { años de trabajo sistemático en publicaciones, además de } \\
\text { muchos otros factores. }\end{array}$ \\
\hline $\begin{array}{l}\text { Factor } \\
\text { de impacto }\end{array}$ & $\begin{array}{l}\text { De este indicador no se identificó un parámetro } \\
\text { local concreto de comparación. De forma precisa } \\
\text { se esperaría que todas las publicaciones tuvieran } \\
\text { FI ya que sólo se valoran a las revistas indizadas, } \\
\text { que en el caso de México es suficiente sin } \\
\text { diferenciar cuartiles. }\end{array}$ & $\begin{array}{l}\text { Este indicador ofrece la limitante que sólo se pueden ofrecer } \\
\text { resultados de publicaciones incluidas en ISI. Los } \mathrm{FI} \text { evaluados } \\
\text { no superan el } 2.000 \text {, en general correspondiendo al tercer y } \\
\text { cuarto cuartil. Se recomienda elevar este indicador a partir de } \\
\text { la búsqueda de publicación en revistas con mayor } \mathrm{FI} \text {. }\end{array}$ \\
\hline $\begin{array}{l}\text { Índice de } \\
\text { inmediatez }\end{array}$ & $\begin{array}{l}\text { De este indicador no se identificó un parámetro } \\
\text { local concreto de comparación. Sin embargo, el } \\
\text { Índice de Inmediatez promedio de las revistas de } \\
\text { química en el período de evaluación fue de } 2.000 \text {. }\end{array}$ & $\begin{array}{l}\text { El Índice de Inmediatez de los artículos evaluados es de } \\
0.384 \text {, resultado bastante alejado del promedio general de la } \\
\text { disciplina. }\end{array}$ \\
\hline
\end{tabular}

Tabla III. Indicadores de impacto

\section{Conclusiones}

A través de la revisión de 15 indicadores, fue factible identificar elementos que contribuyan a definir acciones de mejora, sin embargo, suce- den dos situaciones básicas que pueden propiciar menor utilidad de los datos: la primera, relacionada con la imprecisión normativa que inhibe concordar resultados con indicadores; y la segunda, la carencia de elementos de compara- 